PROCEEDINGS OF THE

AMERICAN MATHEMATICAL SOCIETY

Volume 132, Number 3, Pages 725-734

S 0002-9939(03)07232-0

Article electronically published on October 9, 2003

\title{
HARDY INEQUALITIES RELATED TO GRUSHIN TYPE OPERATORS
}

\author{
LORENZO D'AMBROSIO
}

(Communicated by David S. Tartakoff)

\begin{abstract}
We prove some Hardy type inequalities related to the Grushin type operator $\Delta_{x}+|x|^{2 \gamma} \Delta_{y}$.
\end{abstract}

\section{INTRODUCTION}

The purpose of this paper is to prove Hardy type inequalities related to the degenerate Grushin type operator $\Delta_{\gamma}:=\Delta_{x}+|x|^{2 \gamma} \Delta_{y}\left(x \in \mathbb{R}^{d}, y \in \mathbb{R}^{k}\right)$.

The well-known Hardy inequality for the first-order derivative of a function $u \in$ $\mathscr{C}_{0}^{1}(\Omega)$, where $\Omega$ is an open set of $\mathbb{R}^{n}$, is given by

$$
c \int_{\Omega} \frac{u^{2}}{|x|^{2}} d x \leq \int_{\Omega}|\nabla u|^{2} d x
$$

(see, for instance, 8] and the references therein). Much effort has been made to give explicit values of the constant $c$, and even more, to find its best value $c_{b}$ (see [7, [9]).

The pre-eminent rule of the Hardy inequality in the study of linear and nonlinear partial differential equations is well-known. For instance, let us consider the linear initial value problem

$$
\begin{cases}u_{t}-\Delta u=\lambda \frac{u}{\mid x^{2}}, & \left.x \in \mathbb{R}^{n}, \quad n \geq 3, \quad t \in\right] 0, T[, \quad \lambda \in \mathbb{R}, \\ u(x, 0)=u_{0}(x), & x \in \mathbb{R}^{n}, \quad u_{0} \in L^{2}\left(\mathbb{R}^{n}\right), \quad u_{0}>0 .\end{cases}
$$

Problem (1.2) has a solution if and only if $\lambda \leq\left(\frac{n-2}{2}\right)^{2}=c_{b}$ (see [2] for more details). In recent years this result has been extended in several directions; see, e.g., 3], [4], [1], [13], [18. As an example, consider the following quasilinear problem:

$$
\begin{cases}u_{t}-\operatorname{div}\left(|\nabla u|^{p-2} \nabla u\right)=\lambda \frac{|u|^{p-2} u}{|x|^{p}}, & (x, t) \in \Omega \times] 0, T[, n>p>1, \lambda>0, \\ u(x, 0)=u_{0}(x), & x \in \Omega, \quad u_{0}>0, \\ u(x, t)=0, & x \in \partial \Omega, \quad t \in] 0, T[\end{cases}
$$

where $u_{0}$ satisfies suitable regularity assumptions and $\Omega$ is a bounded domain in $\mathbb{R}^{n}$ containing the origin. In [11, the authors proved that the above problem with $p \geq \frac{2 n}{n+1}$ has a solution if and only if $\lambda \leq c_{n, p}:=\left(\frac{n-p}{p}\right)^{p}$. Again, the result depends

Received by the editors October 18, 2002.

2000 Mathematics Subject Classification. Primary 35H10, 26D10, 46E35.

Key words and phrases. Hardy inequality, Grushin operator.

This research was partially supported by M.U.R.S.T. Programma Nazionale "Metodi Variazionali ed Equazioni Differenziali Nonlineari". 
on the relation between $\lambda$ and the best constant $c_{n, p}$ in the inequality of type (1.1) in $L^{p}$ :

$$
c \int_{\Omega} \frac{|u|^{p}}{|x|^{p}} d x \leq \int_{\Omega}|\nabla u|^{p} d x .
$$

In the Heisenberg group setting, Garofalo and Lanconelli in [12], Niu, Zhang and Wang in [17] and the author in [5] proved, among other results, the following Hardy type inequality related to the Kohn Laplacian operator:

$$
c \int_{\Omega} \frac{u^{2}}{\rho^{2}} \psi^{2} d \xi \leq \int_{\Omega}\left|\nabla_{H} u\right|^{2} d \xi, \quad u \in \mathscr{C}_{0}^{1}(\Omega)
$$

where $\nabla_{H}$ denotes the vector field associated to the Kohn Laplacian $\left(\Delta_{H}=\nabla_{H}\right.$. $\left.\nabla_{H}\right), \rho$ and $\psi$ are, respectively, a suitable distance from the origin and a weight function such that $0 \leq \psi \leq 1$ (for a detailed description of these results we refer the interested reader to [5], [12], [17]).

Recently, in [13, it has been pointed out that the analogue problem of (1.2) involving the Kohn Laplacian $\Delta_{H}$, namely

$$
\begin{cases}u_{t}-\Delta_{H} u=\lambda \psi^{2} \frac{u}{\rho^{2}} & \text { on } \left.\mathbb{R}^{2 n+1} \times\right] 0, T[, \quad \lambda \in \mathbb{R}, \\ u(\cdot, 0)=u_{0}(\cdot) & \text { on } \mathbb{R}^{2 n+1}, \quad u_{0} \in L^{2}\left(\mathbb{R}^{2 n+1}\right), \quad u_{0}>0,\end{cases}
$$

has a positive solution if and only if $\lambda \leq c_{b, H}$, where $c_{b, H}$ is the best constant in (1.4).

Furthermore, an important connection between the Gelfand problem and Hardy inequality has been pointed out in [3] and [4].

Having in mind some extensions of these kinds of results in the setting of secondorder linear degenerate (or singular) partial differential operators, it appears that an important step towards this programme is to establish some fundamental inequalities of Hardy type.

In this paper we shall prove some inequalities of the type (1.1) associated to the Grushin type operator $\Delta_{\gamma}:=\Delta_{x}+|x|^{2 \gamma} \Delta_{y}$ with $\gamma>0\left(x \in \mathbb{R}^{d}, y \in \mathbb{R}^{k}\right)$; see [14], [10] and the references therein.

Let $\nabla_{\gamma}$ be the gradient operator defined by

$$
\nabla_{\gamma}:=\left(\nabla_{x},|x|^{\gamma} \nabla_{y}\right)
$$

We have $\Delta_{\gamma}=\nabla_{\gamma} \cdot \nabla_{\gamma}$.

Our aim is to prove inequalities of the type

$$
c \int_{\Omega}|u|^{p} w^{p} d \xi \leq \int_{\Omega}\left|\nabla_{\gamma} u\right|^{p} d \xi
$$

where $w$ is one of the following functions: $1 /|x|, 1 / \llbracket \xi \rrbracket$ or $|x|^{\gamma} / \llbracket \xi \rrbracket^{1+\gamma}$ and $\llbracket \cdot \rrbracket$ denotes a suitable distance from the origin (see below for the definition). Furthermore, we give an estimate on the optimal constant in (1.5) and in some cases we show its sharp value.

For this goal we shall mainly use a technique developed in [5] and [16]. An interesting outcome of this approach is that, in some cases, one can easily obtain the best constant even for a higher-order generalization of (1.1). We refer to Niu, Zhang and Wang [17] for a different and interesting approach based on the Picone identity [1] in the Heisenberg group setting.

This paper is organized as follows. In the next section, we introduce notation and basic facts about the degenerate operator $\Delta_{\gamma}$. Section 3 is devoted to the proof 
of some Hardy inequalities of type (1.5). In the same section, as a consequence of the technique used in the proof of our main result, we prove a Poincaré inequality related to the operator $\Delta_{\gamma}$.

\section{Notation AND PRELIminary RESUlts}

In this section we shall introduce some notation. Let $\gamma$ be a positive real number and let $\xi=\left(x_{1}, \ldots, x_{d}, y_{1}, \ldots, y_{k}\right)=(x, y) \in \mathbb{R}^{d} \times \mathbb{R}^{k}=\mathbb{R}^{N}$ with $d, k \geq 1$ and $N=d+k$. We denote by $|x|$ (resp. $|y|)$ the Euclidean norm in $\mathbb{R}^{d}\left(\right.$ resp. $\left.\mathbb{R}^{k}\right)$ : $|x|:=\sqrt{x_{1}^{2}+\cdots+x_{d}^{2}}$ (resp. $\left.|y|:=\sqrt{y_{1}^{2}+\cdots+y_{k}^{2}}\right)$. Unless otherwise stated, $\Omega \subset \mathbb{R}^{N}$ will denote an open set.

The symbols div, $\nabla_{\xi}$ and $\Delta_{\xi}$ stand respectively for the usual divergence operator on $\mathbb{R}^{N}$, the gradient and the Laplace operator for functions defined on $\mathbb{R}^{N}$ with respect to the $\xi$-variable.

For $i=1, \ldots, d$, and $j=1, \ldots, k$ consider the vector fields

$$
X_{i}:=\frac{\partial}{\partial x_{i}}, Y_{j}:=|x|^{\gamma} \frac{\partial}{\partial y_{j}},
$$

and the associated gradient as follows:

$$
\nabla_{\gamma}:=\left(X_{1}, \ldots, X_{d}, Y_{1}, \ldots, Y_{k}\right)=\left(\nabla_{x},|x|^{\gamma} \nabla_{y}\right) .
$$

The Grushin operator $\Delta_{\gamma}$ is the operator defined by

$$
\Delta_{\gamma}:=\sum_{i=1}^{d} X_{i}^{2}+\sum_{j=1}^{k} Y_{j}^{2}=\Delta_{x}+|x|^{2 \gamma} \Delta_{y}=\nabla_{\gamma} \cdot \nabla_{\gamma} .
$$

Defining on $\mathbb{R}^{N}$ the dilation $\delta_{\lambda}$ as

$$
\delta_{\lambda}(x, y):=\left(\lambda x, \lambda^{1+\gamma} y\right)
$$

it is not difficult to check that $X_{i}$ and $Y_{i}$ are homogeneous of degree one with respect to the dilation $X_{i}\left(\delta_{\lambda}\right)=\lambda \delta_{\lambda}\left(X_{i}\right), Y_{i}\left(\delta_{\lambda}\right)=\lambda \delta_{\lambda}\left(Y_{i}\right)$, and hence $\nabla_{\gamma}\left(\delta_{\lambda}\right)=\lambda \delta_{\lambda}\left(\nabla_{\gamma}\right)$.

Let $\llbracket \xi \rrbracket=\llbracket(x, y) \rrbracket$ be the following distance from the origin on $\mathbb{R}^{N}$ :

$$
\llbracket \xi \rrbracket=\llbracket(x, y) \rrbracket:=\left(\left(\sum_{i=1}^{d} x_{i}^{2}\right)^{1+\gamma}+(1+\gamma)^{2} \sum_{i=1}^{k} y_{i}^{2}\right)^{\frac{1}{2+2 \gamma}} .
$$

The function $\llbracket \rrbracket$ is related to the fundamental solution at the origin of the Grushin operator $\Delta_{\gamma}$ (see [6]). Furthermore, it is easy to see that $\llbracket \cdot \rrbracket$ is homogeneous of degree one with respect to $\delta_{\lambda}$. Let $R>0$. We shall denote by $B_{R}$ the set

$$
B_{R}:=\left\{\xi \in \mathbb{R}^{N}: \llbracket \xi \rrbracket<R\right\} .
$$

A function $u: \Omega \rightarrow \mathbb{R}$, such that $u(\xi)=u(\llbracket \xi \rrbracket)$ ( $u$ depends only on $\llbracket \xi \rrbracket)$ is said to be radial.

Let $u \in \mathscr{C}^{1}(\Omega)$. If $u$ is radial, then it is easy to check that

$$
\left|\nabla_{\gamma} u(\xi)\right|=\frac{|x|^{\gamma}}{\llbracket \xi \rrbracket^{\gamma}}\left|u^{\prime}(\llbracket \xi \rrbracket)\right| .
$$

Moreover, if $u \in \mathscr{C}^{2}(\Omega)$ and is radial, we find

$$
\Delta_{\gamma} u=\frac{|x|^{2 \gamma}}{\llbracket \xi \rrbracket^{2 \gamma}}\left(u^{\prime \prime}+\frac{d+(1+\gamma) k-1}{\llbracket \xi \rrbracket} u^{\prime}\right) .
$$


Let $\Omega=B_{R_{2}} \backslash \overline{B_{R_{1}}}$, with $0 \leq R_{1}<R_{2} \leq+\infty$ and $u \in \mathscr{C}(\Omega)$. As we shall see below, in some intermediate inequalities appearing in the proof of our main results, we shall need to compute $\int_{\Omega} u$. For this task we can proceed by considering the transformation $\xi:=\Phi\left(\rho, \theta, \theta_{1}, \ldots, \theta_{d-1}, \omega_{1}, \ldots, \omega_{k-1}\right)$, introduced in [6] and defined by

$$
\begin{aligned}
& x_{1}=\rho \sin \theta\left(\sin ^{2} \theta\right)^{-\frac{\gamma}{2(1+\gamma)}} \cos \omega_{1}, \\
& x_{2}=\rho \sin \theta\left(\sin ^{2} \theta\right)^{-\frac{\gamma}{2(1+\gamma)}} \sin \omega_{1} \cos \omega_{2} \text {, } \\
& x_{d-1}=\rho \sin \theta\left(\sin ^{2} \theta\right)^{-\frac{\gamma}{2(1+\gamma)}} \sin \omega_{1} \sin \omega_{2} \ldots \cos \omega_{d-1} \text {, } \\
& x_{d}=\rho \sin \theta\left(\sin ^{2} \theta\right)^{-\frac{\gamma}{2(1+\gamma)} \sin \omega_{1} \sin \omega_{2} \ldots \sin \omega_{d-1},} \\
& y_{1}=\frac{1}{1+\gamma} \rho^{1+\gamma} \cos \theta \cos \theta_{1}, \\
& y_{2}=\frac{1}{1+\gamma} \rho^{1+\gamma} \cos \theta \sin \theta_{1} \cos \theta_{2} \text {, } \\
& y_{k-1}=\frac{1}{1+\gamma} \rho^{1+\gamma} \cos \theta \sin \theta_{1} \sin \theta_{2} \ldots \cos \theta_{k-1}, \\
& y_{k}=\frac{1}{1+\gamma} \rho^{1+\gamma} \cos \theta \sin \theta_{1} \sin \theta_{2} \ldots \sin \theta_{k-1},
\end{aligned}
$$

for $\left.R_{1}<\rho<R_{2}, \theta_{i}, \omega_{j} \in\right] 0, \pi\left[\right.$ for $i=1, \ldots, k-2, j=1, \ldots, d-2, \theta_{k-1}, \omega_{d-1} \in$

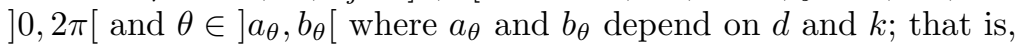

- $\theta \in] 0, \frac{\pi}{2}[$, if $d, k \geq 2$,

- $\theta \in] 0, \pi[$, if $k=1$ and $d \geq 2$,

- $\theta \in]-\frac{\pi}{2}, \frac{\pi}{2}[$, if $d=1$ and $k \geq 2$,

- $\theta \in] 0,2 \pi[$, if $d=k=1$.

Let $J(\Phi)$ be the Jacobian of $\Phi$. By a simple computation we have $|\operatorname{det} J(\Phi)|=$ $\rho^{Q-1} \Theta\left(\theta, \theta_{1}, \ldots, \theta_{d-2}, \omega_{1}, \ldots, \omega_{k-2}\right)$, where $Q:=d+(1+\gamma) k$ and

$$
\Theta:=\left(\frac{1}{1+\gamma}\right)^{k}|\sin \theta|^{\frac{d}{1+\gamma}-1} \cos ^{k-1} \theta \sin ^{k-2} \theta_{1} \ldots \sin \theta_{k-2} \sin ^{d-2} \omega_{1} \ldots \sin \omega_{d-2} .
$$

Therefore, if $u: \Omega \rightarrow \mathbb{R}$ is radial, i.e., $u(\xi)=u(\llbracket \xi \rrbracket)$, then

$$
\int_{\Omega} u(\llbracket \xi \rrbracket) d \xi=s_{n} \int_{R_{1}}^{R_{2}} \rho^{Q-1} u(\rho) d \rho
$$

where

$$
s_{n}:=\int_{a_{\theta}}^{b_{\theta}} d \theta \int_{0}^{\pi} d \theta_{1} \ldots \int_{0}^{\pi} d \theta_{k-2} \int_{0}^{2 \pi} d \theta_{k-1} \int_{0}^{\pi} d \omega_{1} \ldots \int_{0}^{\pi} d \omega_{d-2} \int_{0}^{2 \pi} d \omega_{d-1} \Theta .
$$

Let $|\cdot|$ be the Lebesgue measure on $\mathbb{R}^{N}$. We note that from (2.4) one gets

$$
\left|B_{R}\right|=\left|B_{1}\right| R^{Q}
$$

where $Q:=d+(1+\gamma) k$ is the so-called homogeneous dimension.

Remark 2.1. Using (2.3), we can deduce the following criteria for the integrability of the function $|x|^{p} \llbracket \xi \rrbracket^{q}$ :

i) if $d>-p$ and $Q>-p-q$, then

$$
\int_{B_{1}}|x|^{p} \llbracket \xi \rrbracket^{q} d \xi<+\infty
$$

ii) if $d>-p$ and $Q<-p-q$, then

$$
\int_{\mathbb{R}^{N} \backslash B_{1}}|x|^{p} \llbracket \xi \rrbracket^{q} d \xi<+\infty .
$$


Let $\mathscr{C}_{0}^{k}(\Omega)$ be the set of functions with compact support belonging to $\mathscr{C}^{k}(\Omega)$. Let $p$ be such that $1 \leq p<\infty$. We shall denote by $D_{\gamma}^{1, p}(\Omega)$ the closure of $\mathscr{C}_{0}^{\infty}(\Omega)$ in the norm $\left(\int_{\Omega}\left|\nabla_{\gamma} u\right|^{p} d \xi\right)^{1 / p}$. If $w \in L_{l o c}^{1}(\Omega)$ and $w>0$ a.e. on $\Omega$, then $D_{\gamma}^{1, p}(\Omega, w)$ denotes the closure of $\mathscr{C}_{0}^{\infty}(\Omega)$ in the norm $\left(\int_{\Omega}\left|\nabla_{\gamma} u\right|^{p} w d \xi\right)^{1 / p}$.

\section{HARDY INEQUALITIES}

Throughout this section we shall denote by $\Omega$ an open subset contained in $\mathbb{R}^{N}$. The results of this paper are the following.

Theorem 3.1. Let $p>1, d, k \geq 1$ and $\alpha, \beta \in \mathbb{R}$ be such that $d+(1+\gamma) k>\alpha-\beta-p$ and $d>\gamma p-\beta$. Then, for every $u \in D_{\gamma}^{1, p}\left(\Omega,|x|^{\beta-\gamma p} \llbracket \xi \rrbracket^{(1+\gamma) p-\alpha}\right)$, we have

$$
c_{Q, p, \alpha, \beta}^{p} \int_{\Omega}|u|^{p} \frac{|x|^{\beta}}{\llbracket \xi \rrbracket^{\alpha}} d \xi \leq \int_{\Omega}\left|\nabla_{\gamma} u\right|^{p}|x|^{\beta-\gamma p} \llbracket \xi \rrbracket^{(1+\gamma) p-\alpha} d \xi,
$$

where $c_{Q, p, \alpha, \beta}:=(Q+\beta-\alpha) / p=(d+(1+\gamma) k+\beta-\alpha) / p$.

If $0 \in \Omega$, then the constant $c_{Q, p, \alpha, \beta}^{p}$ in (3.1) is sharp.

In particular, if $Q:=d+(1+\gamma) k>p>1$, then

$$
\begin{aligned}
& \left(\frac{Q-p}{p}\right)^{p} \int_{\Omega} \frac{|u|^{p}}{\llbracket \xi \rrbracket^{p}} \frac{|x|^{\gamma p}}{\llbracket \xi \rrbracket^{\gamma p}} d \xi \leq \int_{\Omega}\left|\nabla_{\gamma} u\right|^{p} d \xi, \quad u \in D_{\gamma}^{1, p}(\Omega), \\
& \left(\frac{Q-p}{p}\right)^{p} \int_{\Omega} \frac{|u|^{p}}{\llbracket \xi \rrbracket^{p}} d \xi \leq \int_{\Omega}\left|\nabla_{\gamma} u\right|^{p} \frac{\llbracket \xi \rrbracket^{\gamma p}}{|x|^{\gamma p}} d \xi, \quad d>\gamma p, u \in D_{\gamma}^{1, p}\left(\Omega, \frac{\llbracket \xi \rrbracket^{\gamma p}}{|x|^{\gamma p}}\right), \\
& \left(\frac{Q-p}{p}\right)^{p} \int_{\Omega} \frac{|u|^{p}}{|x|^{p}} d \xi \leq \int_{\Omega}\left|\nabla_{\gamma} u\right|^{p} \frac{\llbracket \xi \rrbracket^{(1+\gamma) p}}{|x|^{(1+\gamma) p}} d \xi \\
& d>(1+\gamma) p, u \in D_{\gamma}^{1, p}\left(\Omega, \frac{\llbracket \xi \rrbracket^{(1+\gamma) p}}{|x|^{(1+\gamma) p}}\right) .
\end{aligned}
$$

Remark 3.2. If $\gamma=0$, then the operator $\Delta_{\gamma}$ is the standard Laplace operator acting on functions defined on $\mathbb{R}^{N}$ and (3.2) is the classical Hardy inequality (see (1.3)).

Theorem 3.3. Let $d>p>1$. Then, for every $u \in D_{\gamma}^{1, p}(\Omega)$ the inequalities

$$
\begin{aligned}
& b_{d, p}^{p} \int_{\Omega} \frac{|u|^{p}}{|x|^{p}} d \xi \leq \int_{\Omega}\left|\nabla_{\gamma} u\right|^{p} d \xi \\
& b_{d, p}^{p} \int_{\Omega} \frac{|u|^{p}}{\llbracket \xi \rrbracket^{p}} d \xi \leq \int_{\Omega}\left|\nabla_{\gamma} u\right|^{p} d \xi
\end{aligned}
$$

hold with $b_{d, p}:=(d-p) / p$.

In particular, if $p=2$ and $d \geq 3$, we have

$$
\left(\frac{d-2}{2}\right)^{2} \int_{\Omega} \frac{u^{2}}{\llbracket \xi \rrbracket^{2}} d \xi \leq\left(\frac{d-2}{2}\right)^{2} \int_{\Omega} \frac{u^{2}}{|x|^{2}} d \xi \leq \int_{\Omega}\left|\nabla_{\gamma} u\right|^{2} d \xi .
$$

Remark 3.4. From the above results it follows that the best constants in (3.5) and (3.6) lie in $\left[\left(\frac{d-p}{p}\right)^{p},\left(\frac{Q-p}{p}\right)^{p}\right]$.

Before proving our results, we fix some notation. 
Let $\epsilon \geq 0$. Define

$$
\begin{aligned}
r_{\epsilon} & :=\left(\epsilon^{2}+\sum_{i=1}^{d} x_{i}^{2}\right)^{1 / 2}, \\
\sigma_{\epsilon} & :=\left(\begin{array}{cc}
I_{d} & 0 \\
0 & r_{\epsilon}^{\gamma} I_{k}
\end{array}\right) .
\end{aligned}
$$

The symbol $\nabla_{\gamma}^{\epsilon}$ stands for $\sigma_{\epsilon} \nabla$. For any vector field $h \in \mathscr{C}^{1}\left(\Omega, \mathbb{R}^{N}\right)$, we shall write $\operatorname{div}_{\gamma}^{\epsilon}(h):=\operatorname{div}\left(\sigma_{\epsilon} h\right)$.

Clearly, if $\epsilon=0$, then $r_{0}=|x|$ and

$$
\nabla_{\gamma}=\nabla_{\gamma}^{0}=\sigma_{0} \nabla, \quad \Delta_{\gamma}=\operatorname{div}_{\gamma}^{0}\left(\nabla_{\gamma}\right) .
$$

Let $\Omega$ be an open subset of $\mathbb{R}^{N}$ and $\hat{h} \in \mathscr{C}^{1}\left(\Omega, \mathbb{R}^{N}\right)$ be a smooth vector field with compact support. By the divergence theorem we have

$$
\int_{\Omega} \operatorname{div}_{\gamma}^{\epsilon} \hat{h} d \xi=\int_{\partial \Omega} \hat{h} \cdot \sigma_{\epsilon} \nu d \Sigma=0
$$

where $\nu$ denotes the exterior normal at point $\xi \in \partial \Omega$. Choosing $\hat{h}:=|u|^{p} h$ with $p>1, u \in \mathscr{C}_{0}^{1}(\Omega)$ and $h \in \mathscr{C}^{1}\left(\Omega, \mathbb{R}^{N}\right)$, we obtain

$$
\int_{\Omega}|u|^{p} \operatorname{div}_{\gamma}^{\epsilon} h d \xi=-p \int_{\Omega}|u|^{p-2} u \nabla_{\gamma}^{\epsilon} u \cdot h d \xi .
$$

Using the Hölder inequality and (3.7), we can prove the following.

Theorem 3.5. Let $\epsilon \geq 0$ and $h \in \mathscr{C}^{1}\left(\Omega, \mathbb{R}^{2 n+1}\right)$ such that $\operatorname{div}_{\gamma}^{\epsilon} h>0$. Then, for any $p>1$ and $u \in \mathscr{C}_{0}^{1}(\Omega)$, we have

$$
\int_{\Omega}|u|^{p} \operatorname{div}_{\gamma}^{\epsilon} h d \xi \leq p^{p} \int_{\Omega}|h|^{p}\left|\operatorname{div}_{\gamma}^{\epsilon} h\right|^{-(p-1)}\left|\nabla_{\gamma}^{\epsilon} u\right|^{p} d \xi .
$$

The main idea for proving Theorems 3.1 and 3.3 and the Poincaré inequality (3.14) (see Theorem 3.7 below), is to specialize the choice of the vector field $h$ in the inequality (3.8).

Proof of Theorem 3.1. Without loss of generality, we shall consider smooth functions $u \in \mathscr{C}_{0}^{\infty}(\Omega)$. The general case will follow by a density argument.

Let $\epsilon>0$. Define

$$
\rho_{\epsilon}:=\left(r_{\epsilon}^{2+2 \gamma}+(1+\gamma)^{2} \sum_{i=1}^{k} y_{i}^{2}\right)^{\frac{1}{2(1+\gamma)}}
$$

and the vector field $h_{\epsilon}^{1}$ as

$$
h_{\epsilon}^{1}(\xi):=\frac{1}{\rho_{\epsilon}^{\alpha}}\left(\begin{array}{c}
x r_{\epsilon}^{\beta} \\
(1+\gamma) y r_{\epsilon}^{\beta-\gamma-2}|x|^{2}
\end{array}\right) .
$$

A simple computation shows that

$$
\begin{aligned}
\operatorname{div}_{\gamma}^{\epsilon} h_{\epsilon}^{1} & =\operatorname{div} \frac{1}{\rho_{\epsilon}^{\alpha}}\left(\begin{array}{c}
x r_{\epsilon}^{\beta} \\
(1+\gamma) y r_{\epsilon}^{\beta-2}|x|^{2}
\end{array}\right) \\
& =\frac{r_{\epsilon}^{\beta}}{\rho_{\epsilon}^{\alpha}}\left(d+((1+\gamma) k+\beta-\alpha) \frac{|x|^{2}}{r_{\epsilon}^{2}}\right), \\
\left|h_{\epsilon}^{1}\right| & =\frac{r_{\epsilon}^{\beta-\gamma-2}|x|^{2}}{\rho_{\epsilon}^{\alpha}}\left(r_{\epsilon}^{2 \gamma}|x|^{2}+(1+\gamma)^{2}|y|^{2}\right)^{\frac{1}{2}} .
\end{aligned}
$$


Let $f_{\epsilon}(\epsilon>0)$ be defined by $f_{\epsilon}(r):=d+((1+\gamma) k+\beta-\alpha) \frac{r^{2}}{\epsilon^{2}+r^{2}}, r \geq 0$. It is not difficult to see that

$$
f_{\epsilon}(r) \geq \begin{cases}d & \text { if }(1+\gamma) k+\beta-\alpha \geq 0 \\ d+(1+\gamma) k+\beta-\alpha & \text { if }(1+\gamma) k+\beta-\alpha<0\end{cases}
$$

for every $r \geq 0$ and $\epsilon>0$. Since $r_{\epsilon} \geq \epsilon$, if $d+(1+\gamma) k>\alpha-\beta$, it follows that $\operatorname{div}_{\gamma}^{\epsilon} h_{\epsilon}^{1}>0$.

Thus we are in a position to apply Theorem 3.5 to $h=h_{\epsilon}^{1}$. Indeed, from (3.8) we obtain

$$
\begin{aligned}
\int_{\Omega}|u|^{p} \frac{r_{\epsilon}^{\beta}}{\rho_{\epsilon}^{\alpha}} f_{\epsilon}(|x|) d \xi & \\
& \leq p^{p} \int_{\Omega}\left|\nabla_{\gamma}^{\epsilon} u\right|^{p} \frac{|x|^{2 p} r_{\epsilon}^{\beta-2 p-\gamma p}\left(r_{\epsilon}^{2 \gamma}|x|^{2}+(1+\gamma)|y|^{2}\right)^{p / 2}}{\rho_{\epsilon}^{\alpha} f_{\epsilon}(|x|)^{p-1}} d \xi .
\end{aligned}
$$

Let $m:=\min \{d, d+(1+\gamma) k+\beta-\alpha\}>0$. By (3.10) and $r<r_{\epsilon}$, the integrand in the right-hand side of (3.11), can be estimated as follows:

$$
\left|\nabla_{\gamma}^{\epsilon} u\right|^{p} \frac{|x|^{2 p} r_{\epsilon}^{\beta-2 p-\gamma p}\left(r_{\epsilon}^{2 \gamma}|x|^{2}+(1+\gamma)|y|^{2}\right)^{p / 2}}{\rho_{\epsilon}^{\alpha} f_{\epsilon}(|x|)^{p-1}} \leq \frac{\left|\nabla_{\gamma}^{\epsilon} u\right|^{p}}{m^{p-1}} r_{\epsilon}^{\beta-\gamma p} \rho_{\epsilon}^{(1+\gamma) p-\alpha} \in L^{1}(\Omega) .
$$

Therefore, by our assumptions $d+(1+\gamma) k>\alpha-\beta$ and $d>p-\beta$, we can apply the Lebesgue dominated convergence theorem to (3.11), and letting $\epsilon \rightarrow 0$, we obtain the claim.

The choices $(\alpha, \beta)=((1+\gamma) p, \gamma p),(\alpha, \beta)=(p, 0)$ and $(\alpha, \beta)=(0,-p)$, in (3.1), yield the inequalities (3.2), (3.3) and 3.4 respectively.

It remains to show that the constant $c_{Q, p, \alpha, \beta}^{p}$ appearing in (3.1) is sharp.

First we consider the case $\Omega=\mathbb{R}^{N}$. In doing so we shall adapt the original idea of Hardy (see [15]) for the one-dimensional Euclidean case.

Given $\epsilon>0$, consider the function

$$
u(\rho):= \begin{cases}C_{\epsilon} & \text { if } \rho \in[0,1], \\ C_{\epsilon} \rho^{-c_{Q, p, \alpha, \beta}-\epsilon} & \text { if } \rho>1,\end{cases}
$$

where $C_{\epsilon}:=\left(c_{Q, p, \alpha, \beta}+\epsilon\right)^{-1}$. We have

$$
u^{\prime}(\rho)= \begin{cases}0 & \text { if } \rho \in] 0,1[, \\ -\rho^{-\frac{Q+\beta-\alpha+p}{p}-\epsilon} & \text { if } \rho>1,\end{cases}
$$

and by computation

$$
\begin{aligned}
& \int_{\mathbb{R}^{N}}|u(\llbracket \xi \rrbracket)|^{p} \frac{|x|^{\beta}}{\llbracket \xi \rrbracket^{\alpha}} d \xi=C_{\epsilon}^{p}\left(\int_{B_{1}} \frac{|x|^{\beta}}{\llbracket \xi \rrbracket^{\alpha}} d \xi+\int_{\mathbb{R}^{N} \backslash B_{1}}|x|^{\beta} \llbracket \xi \rrbracket^{-Q-\beta-\epsilon p} d \xi\right) \\
& =C_{\epsilon}^{p}\left(\int_{B_{1}} \frac{|x|^{\beta}}{\llbracket \xi \rrbracket^{\alpha}} d \xi\right. \\
& \left.+\int_{\mathbb{R}^{N} \backslash B_{1}} \frac{|x|^{\gamma p}}{\llbracket \xi \rrbracket^{\gamma p}}\left|\llbracket \xi \rrbracket^{-\frac{Q+\beta-\alpha+p}{p}-\epsilon}\right| p|x|^{\beta-\gamma p} \llbracket \xi \rrbracket^{(1+\gamma) p-\alpha} d \xi\right) \\
& =C_{\epsilon}^{p}\left(\int_{B_{1}} \frac{|x|^{\beta}}{\llbracket \xi \rrbracket^{\alpha}} d \xi+\int_{\mathbb{R}^{N}}\left|\nabla_{\gamma} u\right|^{p}|x|^{\beta-\gamma p} \llbracket \xi \rrbracket^{(1+\gamma) p-\alpha} d \xi\right) \text {, }
\end{aligned}
$$


where, in the last identity, we have used the relation (2.2) and the fact that $u^{\prime}$ vanish on $B_{1}$. Since the addenda in the right-hand side of (3.12) are integrable (see Remark [2.1), by letting $\epsilon \rightarrow 0$, we easily get the claim.

In order to conclude in the general case, we proceed as follows: let $c_{b}(\Omega)$ be the best constant in (3.1). By invariance of (3.1) under the dilation $\delta_{\lambda}$ we have

$$
c_{b}\left(B_{R}\right)=c_{b}\left(B_{1}\right) \text { for any } R>0 .
$$

Furthermore, we note that, if $B_{R} \subset \Omega \subset \mathbb{R}^{N}$, then

$$
c_{Q, p, \alpha, \beta}^{p}=c_{b}\left(\mathbb{R}^{N}\right) \leq c_{b}(\Omega) \leq c_{b}\left(B_{R}\right)=c_{b}\left(B_{1}\right) .
$$

Let $\varphi \in \mathscr{C}_{0}^{\infty}\left(\mathbb{R}^{N}\right)$. Since the support of $\varphi$ is compact, then (3.1) holds for $\varphi$ with $\Omega=B_{R}, R$ large enough and $c=c_{b}\left(B_{R}\right)=c_{b}\left(B_{1}\right)$. Therefore, $c_{b}\left(B_{1}\right) \leq c_{b}\left(\mathbb{R}^{N}\right)$ and from (3.13) we conclude the proof.

Remark 3.6. Let $\phi \in \mathscr{C}^{2}(\Omega)$ and $\epsilon=0$. Setting $h:=\nabla_{\gamma} \phi$, we see that Theorem 3.3 can be reformulated as follows: if $\Delta_{\gamma} \phi>0$, then for any $p>1$ and $u \in \mathscr{C}_{0}^{1}(\Omega)$ we have

$$
\int_{\Omega}|u|^{p} \Delta_{\gamma} \phi d \xi \leq p^{p} \int_{\Omega}\left|\nabla_{\gamma} \phi\right|^{p}\left|\Delta_{\gamma} \phi\right|^{-(p-1)}\left|\nabla_{\gamma} u\right|^{p} d \xi .
$$

Following this idea, one can easily realize that the main problem is to decide whether such a function exists. Indeed, via a standard regularization argument, we see that (3.2) can be proved if there exists $\phi \in \mathscr{C}^{2}(\Omega)$ such that $\nabla_{\gamma} \phi=h_{0}^{1}$, where $h_{0}^{1}$ is the vector field defined in (3.9) with $\epsilon=0, \alpha=(1+\gamma) p$ and $\beta=\gamma p$. A simple computation shows that this is possible only when $\gamma=0$ or $p=2$.

In the case $\gamma=0$ and $p>1$, we obtain the classical Hardy inequality associated to the $p$-Laplacian operator on $\mathbb{R}^{N}$ (see [9]), while if $\gamma>0$ and $p=2$, the function $\phi$ is given by $\phi(\xi):=\ln \llbracket \xi \rrbracket$.

This show that in the framework of Hardy type inequalities associated to secondorder elliptic operators with degenerate or singular coefficients, the method based on the choice of a general vector field is more efficient.

Proof of Theorem [3.3] Define $h_{\epsilon}^{2}$ as follows:

$$
h_{\epsilon}^{2}(\xi):=\frac{1}{r_{\epsilon}^{p}}\left(\begin{array}{l}
x \\
0
\end{array}\right)
$$

We have

$$
\begin{aligned}
\operatorname{div}_{\gamma}^{\epsilon} h_{\epsilon}^{2} & =\frac{1}{r_{\epsilon}^{p}}\left(d-p \frac{|x|^{2}}{r_{\epsilon}^{2}}\right), \\
\left|h_{\epsilon}^{2}\right| & =\frac{|x|}{r_{\epsilon}^{p}} .
\end{aligned}
$$

Since $d>p$, we get $\operatorname{div}_{\gamma}^{\epsilon} h_{\epsilon}^{2}>0$, and, as in the previous case, by (3.8) it follows that

$$
\int_{\Omega} \frac{|u|^{p}}{r_{\epsilon}^{p}}\left(d-p \frac{|x|^{2}}{r_{\epsilon}^{2}}\right) d \xi \leq p^{p} \int_{\Omega} \frac{|x|^{p}}{r_{\epsilon}^{p}\left(d-p \frac{|x|^{2}}{r_{\epsilon}^{2}}\right)^{p-1}}\left|\nabla_{\gamma}^{\epsilon} u\right|^{p} d \xi
$$

By the Lebesgue dominated convergence theorem, the last inequality implies (3.5). Finally, using the fact that $\llbracket \xi \rrbracket \geq|x|$ and (3.5), we obtain (3.6). This concludes the proof.

We note that (3.8) also yields a Poincaré inequality for the vector field $\nabla_{\gamma}$ on domains $\Omega$ contained in a slab. More precisely, we have 
Theorem 3.7. Let $\Omega$ be an open subset on $\mathbb{R}^{N}$. Suppose that there exist $R>0$, a real number $s$ and an integer $1 \leq j \leq d$ such that for any $\xi=(x, y) \in \Omega$, it follows that $\left|x_{j}-s\right| \leq R$.

Then, for every $u \in \mathscr{C}_{0}^{1}(\Omega)$, we have

$$
c \int_{\Omega}|u|^{p} d \xi \leq \int_{\Omega}\left|\nabla_{\gamma} u\right|^{p} d \xi
$$

where $c=\left(\frac{1}{p R}\right)^{p}$.

The proof of the theorem follows from Theorem 3.5 by using the vector field defined by

$$
h:=\left(\begin{array}{c}
0 \\
x_{j}-s \\
0
\end{array}\right) .
$$

\section{ACKNOWLEDGMENT}

The author thanks professor Enzo Mitidieri for many stimulating conversations.

\section{REFERENCES}

1. W. Allegretto and Y. X. Huang, A Picone's identity for the p-Laplacian and applications, Nonlinear Anal. 32 (1998), 819-830. MR 99c:35051

2. P. Baras and J. A. Goldstein, The heat equation with a singular potential, Trans. Amer. Math. Soc. 284 (1984), 121-139. MR 85f:35099

3. H. Brezis and X. Cabré, Some Simple Nonlinear PDE's without Solutions, Boll. Unione Mat. Ital. Sez. B Artic. Ric. Mat. 1 (1998), 223-262. MR 99j:35001

4. H. Brezis and J. L. Vázquez, Blow-up solutions of some nonlinear elliptic problem, Rev. Mat. Univ. Complut. Madrid 10 (1997), no. 2, 443-469. MR 99a:35081

5. L. D'Ambrosio, Some Hardy Inequalities on the Heisenberg Group, Diff. Equations, to appear.

6. L. D'Ambrosio and S. Lucente, Nonlinear Liouville Theorems for Grushin and Tricomi Operators, J. Differential Equations 193 (2003), no. 2, 511-541.

7. E. B. Davies, The Hardy constant, Quart. J. Math. Oxford Ser. 46 (1995), 417-431. MR 97b:46041

8. E. B. Davies, A review of Hardy inequalities, in Operator Theory: Advances and Applications, Vol. 110 (1999), Birkhäuser-Verlag Basel, 55-67. MR 2001f:35166

9. E. B. Davies and A. M. Hinz, Explicit constants for Rellich inequalities in $L_{p}(\Omega)$, Math. Z. 227 (1998), 511-523. MR 99e:58169

10. B. Franchi, C. E. Gutiérrez, and R. L. Wheeden, Weighted Sobolev-Poincaré inequalities for Grushin type operators, Comm. Partial Differential Equations 19 (1994), 523-604. MR 96h:26019

11. J. P. García Azorero and I. Peral Alonso, Hardy Inequalities and Some Critical Elliptic and Parabolic Problems, J. Differential Equations 144 (1998), 441-476. MR 99f:35099

12. N. Garofalo and E. Lanconelli, Frequency Functions on the Heisenberg Group, the Uncertainty Principle and Unique Continuation, Ann. Inst. Fourier, Grenoble 40 (1990), 313-356. MR 91i:22014

13. J. A. Goldstein and Qi S. Zhang, On a Degenerate Heat Equation with a Singular Potential, J. Funct. Anal. 186 (2001), 342-359. MR 2002k:35179

14. V. V. Grushin, On a Class of Hypoelliptic Operators, Math. USSR Sbornik 12 (1970), 458476.

15. G. H. Hardy, J. E. Littlewood, and G. Polya, Inequalities, Cambridge University Press, UK, 1934.

16. E. Mitidieri, A simple approach to Hardy inequalities, Mat. Zametki 67 (2000), 563-572. MR 2001f: 26022

17. P. Niu, H. Zhang, and Y. Wang, Hardy Type and Rellich Type Inequalities on the Heisenberg Group, Proc. Amer. Math. Soc. 129 (2001), 3623-3630. MR 2002i:26009 
18. J. L. Vazquez and E. Zuazua, The Hardy Inequality and the Asymptotic Behavior of the Heat Equation with an Inverse-Square Potential, J. Funct. Anal. 173 (2000), 103-153. MR 2001j:35122

SiSSA-ISAS, v. Beirut, $2-4-34014$, Trieste, Italy

Dipartimento di Matematica, via Orabona, 4 - I-70125, Bari, Italy

E-mail address: dambros@dm.uniba.it 